\title{
Radical Salvage Prostatectomy with Pelvic Lymphadenectomy Extended Post Primary Treatment with Prostate Radiotherapy - Case Report and Literature Review
}

\author{
Daniel Savoldi Juraski, MD; Rodrigo Galves Mesquita Martins, MD; Diogo Eugenio Abreu da Silva, MsC; Tomás \\ Accioly de Souza, MD and José Anacleto Dutra de Resende Junior, PhD* \\ Department of Urology, Lagoa Federal Hospital, Brazil \\ *Corresponding author: José Anacleto Dutra de Resende Junior, Department of Urology, Lagoa Federal Hospital, Rua Jardim Botânico 501-6ªndar, \\ Jardim Botânico, Rio de Janeiro, Brazil
}

Submission: 笽October 08, 2017; Published: 㘹January 31, 2018

\begin{abstract}
Patient male 65 years old (2017), submitted in July 2009 to prostatic radiotherapy without hormonal blockade due to prostate adenocarcinoma Gleason $6(3+3)$ with total initial PSA of 43.67 and bone scintigraphy (2009) not identifying metastatic disease. At follow-up, it maintained high total PSA (16.25 in December 2014). Recurrence tests (MRI, bone scintigraphy, and total PSA) excluded lymph node and metastatic disease, but with increasing total PSA. The patient was submitted to prostate biopsy identifying a prostate adenocarcinoma Gleason $7(4+3)$ and surgical salvage treatment was indicated. In July 2016, he underwent radical salvage prostatectomy with extended pelvic lymphadenectomy and was diagnosed a prostate adenocarcinoma Gleason $9(4+5)$, involving both prostatic lobes with a right border, focally compromised by neoplasia, bladder border with atypical cells, neoplasia-free urethral limit, seminal vesicles and deferent neoplasia-free, neoplastic-free pelvic lymph nodes with pathological staging: pT2c pN0. Postoperative follow-up (7 months after surgery) maintained an undetectable total PSA ( $<0.09)$, continent and with erection in use of phosphodiesterase type 5 inhibitors.
\end{abstract}

Keywords: Radical salvage prostatectomy; Pelvic lymphadenectomy; Prostatic radiation therapy

\section{Introduction}

Salvage prostatectomy is a plausible option for patients treated primarily with prostate cancer radiotherapy for prostate cancer who have progressed with biochemical relapse and who do not have metastatic disease. The main factors that disadvantage this procedure are the clinical conditions of the patient, the PSA levels and the findings in prostate biopsy after radiotherapy, making its indication more restricted and its use rarer. The present article reports a case of a patient submitted to radical salvage prostatectomy with pelvic lymphadenectomy extended post primary treatment with prostatic radiotherapy in our service and a brief review of the literature.

\section{Clinical Findings and Diagnostic Assessment}

Male patient, now aged 65 years (2017), referred to our service in April 2015, aged 62, already diagnosed with prostate cancer in 2009 by prostate biopsy with histopathological report identifying a Gleason 6 adenocarcinoma (3+3), Initial total PSA of 43.67 and bone scintigraphy (2009) excluding metastatic disease. He was submitted to prostatic bed radiotherapy in July 2009 (total of 70 Gy) without adjuvant androgenic block. In the first consultation
(April 2015), the patient had the last total PSA of December 2014 of 16.25 and the digital prostate examination had a moderate increase (grade II) and without the presence of nodulations or irregularities. As for the previous pathological history, he had diabetes mellitus using oral hypoglycemic drugs only, denying other diseases or previous surgeries. His occupation was a mason. New exams (nuclear magnetic resonance of pelvis, bone scintigraphy and total PSA) were requested.

On return, nuclear magnetic resonance of pelvis (July 2015) showed a 36 gram prostate with a small oval image of about $0.9 \mathrm{~cm}$, without contrast enhancement, in the peripheral zone, but without pelvic lymph node enlargement or hydronephrosis. The total PSA for May 2015 was 16.75, December 2015 was 18.6 and March 2016 was 27.8. Bone scintigraphy of April 2016 excluded the possibility of metastatic disease. Prostate biopsy was indicated due to the findings. The histopathological report of prostate biopsy performed in April 2016 showed a prostate adenocarcinoma Gleason $7(4+3)$ in $20 \%$ of the left lobe samples only. Salvage radical prostatectomy was then indicated with extended pelvic lymphadenectomy and requested exams of preoperatively 


\section{Therapeutic Intervention}

The patient was fit for the procedure and underwent radical prostatectomy with pelvic lymphadenectomy extended in July 2016

(Figure 1).

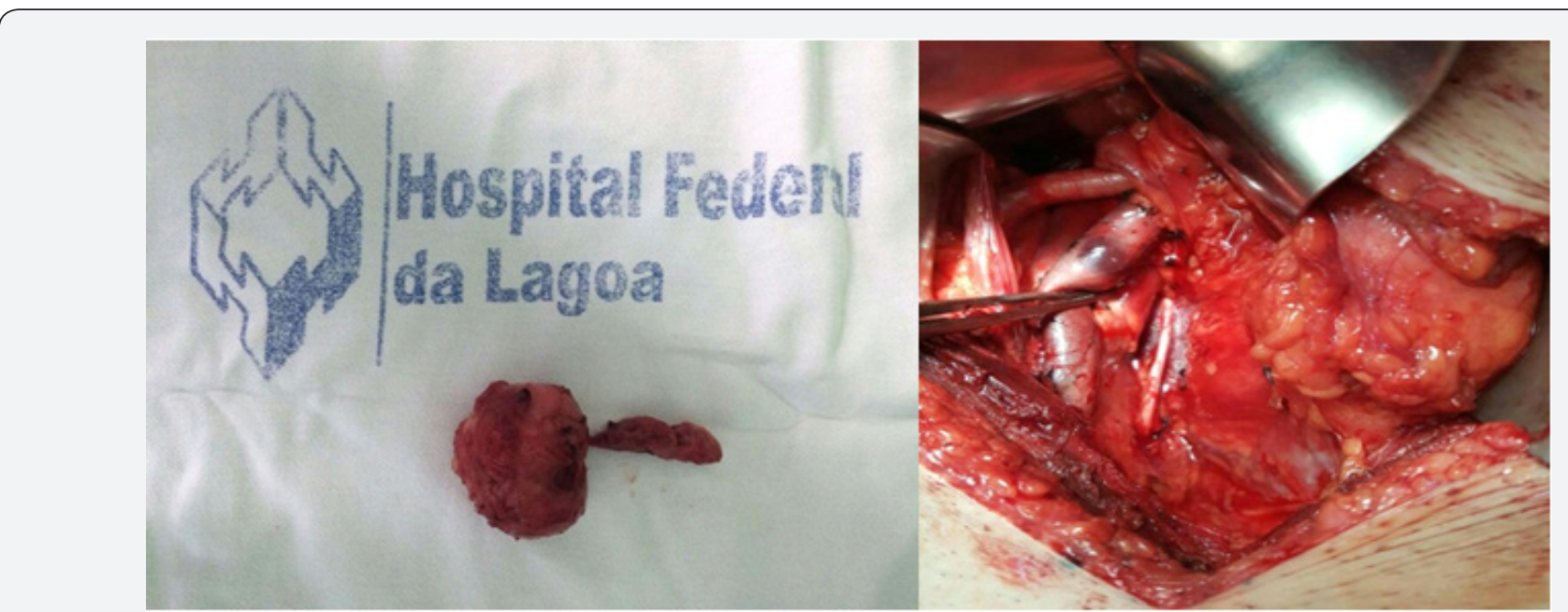

Figure 1: Radical prostatectomy product and extended pelvic lymphadenectomy.

\section{Clinical Follow-Up and Results}

The histopathological report of the surgical specimen and of the pelvic lymph nodes revealed a prostate adenocarcinoma Gleason $9(4+5)$ affecting both prostate lobes with a surgical border on the right focally compromised by neoplasia, bladder border with atypical cells, neoplastic free urethral limit, vesicles seminal and deferent neoplasms, pelvic lymph nodes free of neoplasia with pathological staging: pT2c pN0.

At the postoperative follow-up, the total PSA levels in August 2016 were $<0.09$, October 2016 was $<0.05$ and February 2017 was $<0.05$. In the last consultation in February 2017, the patient was continent and presented with erection with phosphodiesterase type 5 inhibitors.

\section{Discussion}

Patients undergoing radical salvage prostatectomy can benefit significantly. The biochemical recurrence-free survival rate at 5 years and at 10 years may reach $47-82 \%$ and $28-53 \%$, respectively, and specific cancer survival and overall survival of $70-83 \%$ and $54-89 \%$ respectively [1]. The best results are obtained in patients with organ-confined disease compared to locally advanced disease with disease-free survival of $70-80 \%$ and $40-60 \%$, respectively [2]. Predictors of better prognosis are patients with a life expectancy greater than 10 years, total PSA pre-salvage prostatectomy $<10 \mathrm{ng} /$ $\mathrm{dl}$, Gleason score $\leq 7$ before salvage prostatectomy biopsy, absence of lymph node involvement, and patients with clinical staging initial T1 or T2 [1]. Despite the oncological results obtained, this procedure compared to open radical prostatectomy for primary treatment has higher rates of urinary incontinence, erectile dysfunction, anastomosis stenosis, urinary retention, urinary fistula, abscess and rectum lesion [1,3,4].

\section{Conclusion}

The radical salvage prostatectomy is a good option for patients with organ-confined disease and who have good clinical conditions to undergo the procedure. However, due to a lower number of patients under these specific conditions, their use becomes more restricted and rare. In addition to being physically fit, the patient should be aware of the risks related to the procedure and the perioperative and postoperative complications. A good doctorpatient relationship is very important.

\section{References}

1. Chade DC, Eastham J, Graefen M, Hu JC, Karnes RJ, et al. (2012) Cancer control and functional outcomes of salvage radical prostatectomy for radiation-recurrent prostate cancer: a systematic review of the literature. Eur Urol 61(5): 961-971.

2. Heidenreich A, Richter S, Thüer D, Pfister D (2010) Prognostic parameters, complications, and oncologic and functional outcome of salvage radical prostatectomy for locally recurrent prostate cancer after $21^{\text {st }}$ century radiotherapy. Eur Urol 57(3): 437-443.

3. Stephenson AJ, Scardino PT, Bianco FJJr, DiBlasio CJ, Fearn PA, etal. (2004) Morbidity and functional outcomes of salvage radical prostatectomy for locally recurrent prostate cancer after radiation therapy. J Urol 172(6): 2239-2243.

4. Gotto GT, Yunis LH, Vora K, Eastham JA, Scardino PT, et al. (2010) Impact of prior prostate radiation on complications after radical prostatectomy. J Urol 184(1): 136-142. 